\title{
Thermomechanical Behaviour of a PWB by Speckle Interferometry Technique
}

\author{
Bartolomeo Trentadue and Giuseppe Illuzzi \\ Dipartimento di Meccanica, Matematica e Management, Politecnico di Bari, Viale Japigia 182, 70126 Bari, Italy \\ Correspondence should be addressed to Bartolomeo Trentadue; btrentadue@poliba.it
}

Received 4 June 2015; Accepted 30 July 2015

Academic Editor: Guangya Zhou

Copyright ( $) 2015$ B. Trentadue and G. Illuzzi. This is an open access article distributed under the Creative Commons Attribution License, which permits unrestricted use, distribution, and reproduction in any medium, provided the original work is properly cited.

The speckle interferometry technique has been used in this work in order to determine the thermomechanical behaviour of Printed Wiring Board (PWB) (circuits) of a radio integrated with tape player and speakers. A preliminary experiment of such technique has been carried out on a single electronic component (silicon transistor), during the thermal transient and at the steady state. The thermal deformation and stresses on PWB have been obtained through related experimental analyses on both cases. The results showed a very good applicability of speckle technique on the irregular object surface as PWB.

\section{Introduction}

Stresses in electronic packages can cause premature mechanical failures of their components: fracture of the die, severing of connections, die bond failure, solder and components fatigue failure, and so forth. These stresses are often thermally induced and result from uneven expansions and contractions of various assembly materials due to mismatches in the coefficients of thermal expansion [1-3].

Many experimental and numerical analyses have been carried out on electronic packages. In particular, the research has been concentrated on the local strains and stresses of solder joints. This research has been aimed at achieving the optical measurement on the Printed Wiring Board of a radio integrated with tape player and speakers, considering that PWB can deform under the moments and forces anchoring the thermally stressed solder joints. On the other hand, even though the overstress of boards is rare, the circuits lines running on the surface should be stress checked.

Many available full-field and noncontact optical techniques, such as Moiré and its variations, Moiré Interferometry, have been used for this kind of problems [3]. However, most of these methods need a good flatness of object surface for the replication of gratings. Considering the applicability limits for each technique in relation to the model being analysed, the speckle techniques have been shown undoubtedly to be the unique method for our purpose, which has been also verified by the authors' experience on a silicon transistor during the thermal transient and the steady state [4].

\section{Speckle Interferometry}

Speckle interferometry relies on the cyclic correlation analyses of speckle patterns for different loading states. It can be carried out in real time as electronic speckle pattern interferometry (ESPI) or digital speckle pattern interferometry (DSPI), respectively, if speckle patterns of the object surface obtained through a CCD-camera at different loads are analogically or digitally subtracted [5-10].

By means of the speckle technique shown in Figure 1, it was possible to obtain the in-plane displacement fields of the specimen subject to mechanical and thermal loads. Displacement components $u(x, y)$ and $v(x, y)$ are measured at each pixel $(x, y)$. Such displacement fields are represented by the speckle fringes with bright and dark zones.

For the bright zones, the following holds:

$$
u=\frac{(2 N+1) \lambda}{4 \sin \theta}
$$

where $N$ is the fringe order, $\theta$ is the illumination angle, and $\lambda$ is the wavelength of laser beam. 

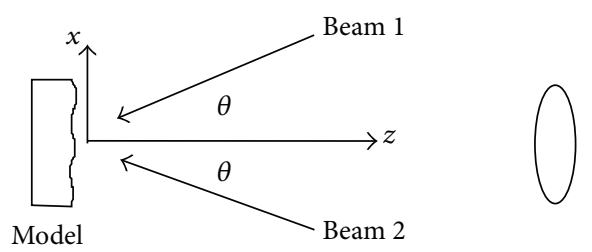

FIGURE 1: In-plane speckle interferometry.

For the dark zones (which is also called the correlated zones), the following holds:

$$
u=\frac{N \lambda}{2 \sin \theta}
$$

By the displacement obtained, the strains of each point in the measuring field can be determined by

$$
\begin{aligned}
\varepsilon_{x x} & =\frac{\partial u(x, y)}{\partial x}, \\
\varepsilon_{y y} & =\frac{\partial v(x, y)}{\partial y}, \\
\gamma_{x y} & =\frac{\partial v(x, y)}{\partial x}+\frac{\partial u(x, y)}{\partial y} .
\end{aligned}
$$

By the Hooke law, the stresses have the following calculations:

$$
\begin{aligned}
\sigma_{x x} & =\frac{E}{(1+v)(1-2 v)}\left[(1-v) \varepsilon_{x x}+v \varepsilon_{y y}\right] ; \\
\sigma_{y y} & =\frac{E}{(1+v)(1-2 v)}\left[(1-v) \varepsilon_{y y}+v \varepsilon_{x x}\right] ; \\
\tau_{x y} & =G \gamma_{x y}=\frac{E}{2(1+v)} \gamma_{x y},
\end{aligned}
$$

where $E$ is Young's modulus, $G$ is shear modulus, and $v$ is Poisson ratio.

The whole field fringe analyses can be accomplished by simple manually curve drawing or by recently developed automatic fringe analysis methods such as Fourier transform and phase-shifting.

The benefits in using speckle interferometry in the PWB analyses are

(1) the possibility of carrying out the test in real time and following thermal transient processes;

(2) nonmandatory requirement of surface flatness under measurement as in the other methods, for example, Moiré Interferometry, where a good plane surface is necessary for the replication of grating;

(3) solder joints in the backside of PWB that may also have the speckle distribution; however in other experimental techniques they are considered pure defects.

Automatic fringe analysis methods [10-14] have enforced greatly the application of speckle methods overcoming its shortcoming of few fringes and low contrast.

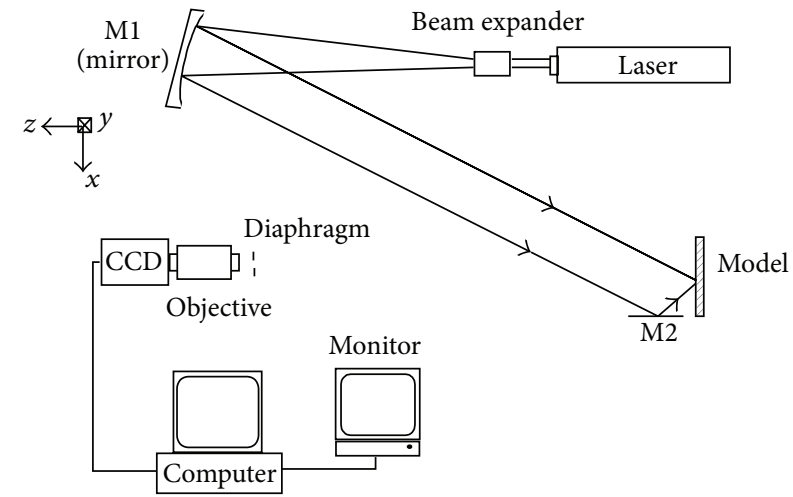

FIgURE 2: Experimental set-up.

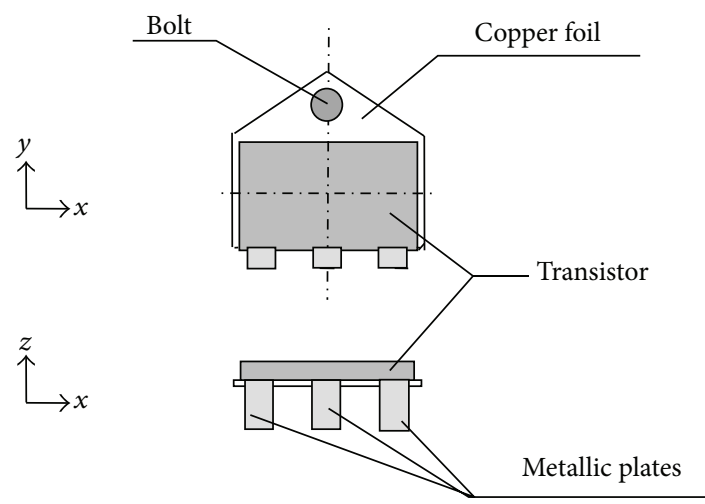

Figure 3: Transistor scheme.

\section{Experimental Set-Up}

The optical set-up is shown in Figure 2. A $35 \mathrm{~mW}$ He-Ne laser of wavelength $(\lambda) 632.8 \mathrm{~nm}$ was utilized for this application. A parabolic mirror M1 is used as reflector and collimator.

A spatial filter (pinhole) of $10 \mu \mathrm{m}$ was placed after the beam expander in order to ensure a good quality of speckle intensity pattern. The mirror M2 was positioned perpendicular to the specimen surface to obtain a symmetric incident beam with that coming directly from the mirror M1 at angle $\theta$. The objective diaphragm was used to control the dimension and form of speckle patterns. The speckle patterns were recorded and processed through CCD-camera to an "Image Processing System."

\section{Experiments on a Silicon Transistor}

A transistor (Figure 3) was tested first in order to analyze the effect of the constraint points on the wiring board. The transistor was considered to be subjected to a plane strain field (shown in Figure 4(a)). Each point has two displacement components in the plane: $u(x, y)$ in the $x$ direction and $v(x, y)$ in the $y$ direction.

The fringe analysis gives directly the displacements, so that it is possible to calculate the strains without considering the analytical solution of $u(x, y)$ and $v(x, y)$. The two displacement components were figured out analyzing two 


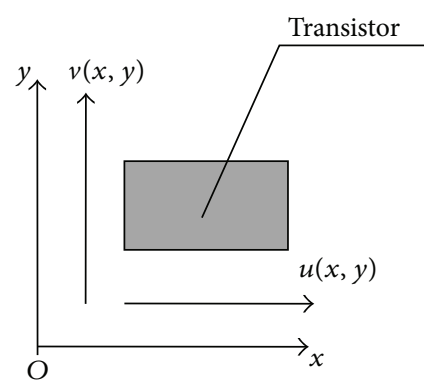

(a)

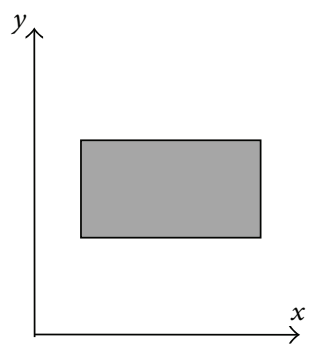

(b)

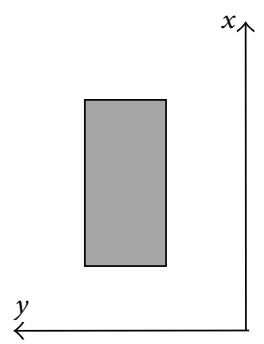

(c)

FIGURE 4: Displacements and positions of the transistor in the test: (a) displacements; (b) horizontal test; (c) vertical test.

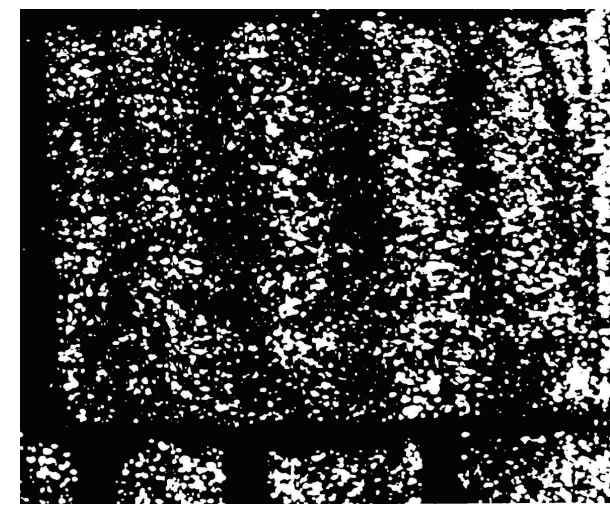

(a)

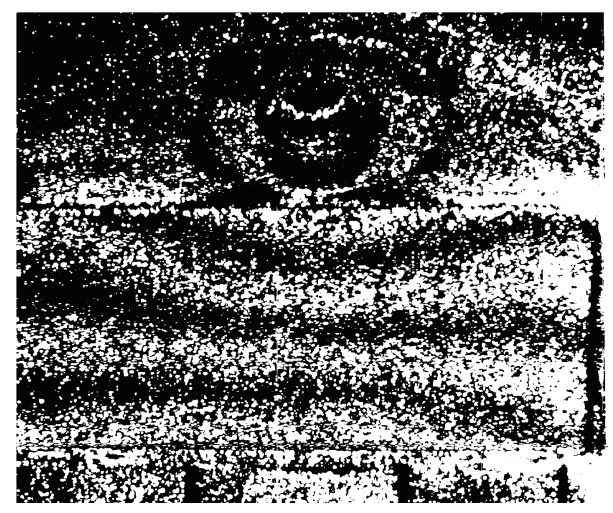

(b)

FIGURE 5: Correlation fringes: (a) horizontal displacement; (b) vertical displacement.

different tests: one with the specimen in horizontal position (Figure $4(\mathrm{~b})$ ) and the other, after a rotation of $90^{\circ}$, in vertical position (Figure $4(\mathrm{c})$ ). Several measurements were carried out for both tests during the transient state and at the steady state.

Figure 5 shows the speckle fringe patterns of horizontal and vertical displacements, respectively. The transistor has the dimensions of $L_{x}=14.7 \mathrm{~mm}$ and $L_{y}=10 \mathrm{~mm}$. Considering the He-Ne laser wavelength $\lambda=632.8 \mathrm{~nm}$ and the beam incident angle $\theta=40^{\circ}$, it is possible to determine the average elongation $\left(\Delta L_{x}, \Delta L_{y}\right)$ for the whole transistor surface due to the thermal load:

$$
\begin{aligned}
& \Delta L_{x}=\frac{N_{x} \lambda}{2 \sin \vartheta} ; \\
& \Delta L_{y}=\frac{N_{y} \lambda}{2 \sin \vartheta},
\end{aligned}
$$

where $N_{x}$ and $N_{y}$ represent the fringe number in the $x$ and $y$ directions, respectively. Thus, the average strains can also be calculated by

$$
\begin{aligned}
& \varepsilon_{x x}=\frac{\Delta L_{x}}{L_{x}} ; \\
& \varepsilon_{y y}=\frac{\Delta L_{y}}{L_{y}} .
\end{aligned}
$$

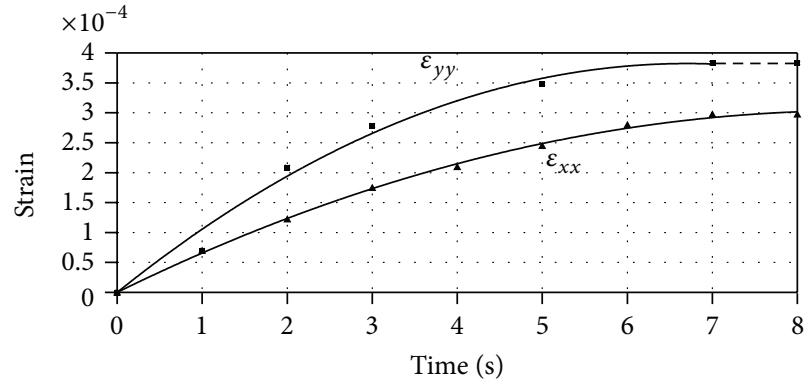

Figure 6: Average strain developed in the transistor with the time.

Figure 6 gives the average strain in the function of the time for both directions. The average $\varepsilon_{x x}$ and $\varepsilon_{y y}$ at steady state were calculated as $2.97 \times 10^{-4}$ and $3.83 \times 10^{-4}$, respectively. The strain in the $y$ direction is considerably higher due to the different level of kinematic constraints deriving from the soldering process.

The local strains $\varepsilon_{x x}=\partial u / \partial x$ and $\varepsilon_{y y}=\partial v / \partial y$ in the sections $(a-a)$ and $(b-b)$ shown in Figure 7 were determined. The strain and stress distribution [2] along section $(a-a)$ are given in Figure 8.

The $\sigma_{y y}$ value decreases while moving to the edges of the transistor. In the diagram shown in Figure 8, a small increment was observed at the location close to the other two 


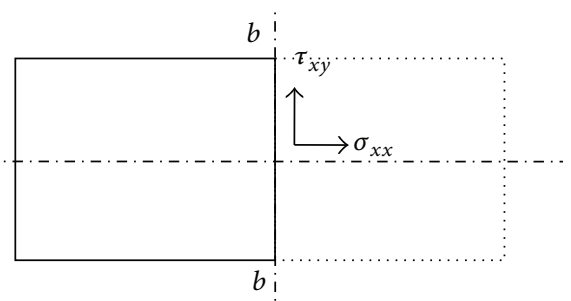

(a)

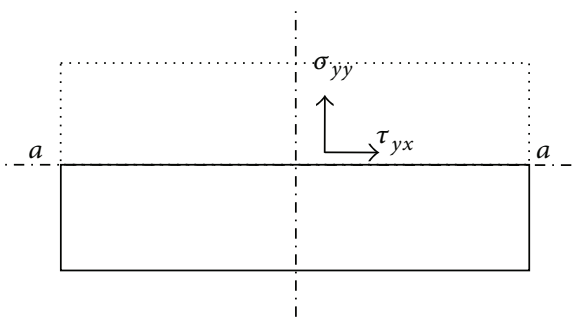

(b)

FIGURE 7: Transistor sections analyzed in the ESPI measurements.

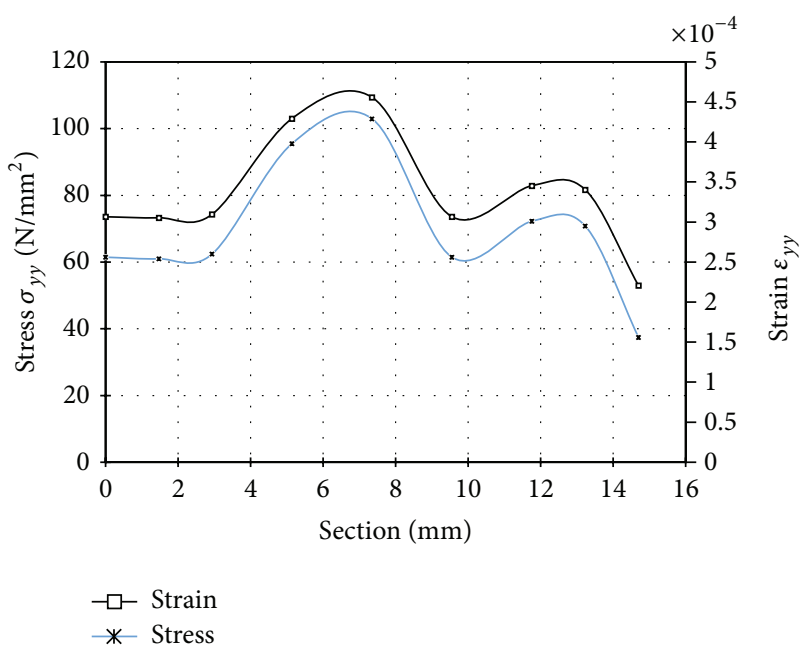

FIGURE 8: Strain $\varepsilon_{y y}$ and stress $\sigma_{y y}$ distribution along $(a-a)$ section.

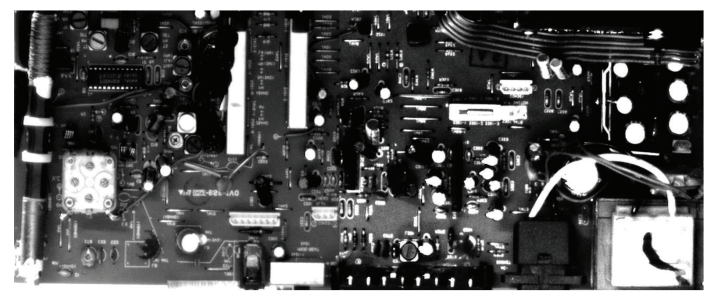

Figure 9: The PWB of a radio and its supported components.

constraints (metallic welded plates). The maximum $\sigma_{y y}$ value was found to be 109.4 MPa.

\section{Measurements on a Printed Wiring Board}

A Printed Wiring Board (PWB) of a radio integrated with tape player and speakers has been taken under experiments (Figure 9). On the backside of the board, the speckle interferometry technique has been applied. The natural surface of PWB consisted of a substrate of polyimide material covered with printed circuit lines and solder joints pattern. In order to obtain a good quality of speckle pattern, the surface was coated with an insulated silver spray, which can be seen in Figure 10.

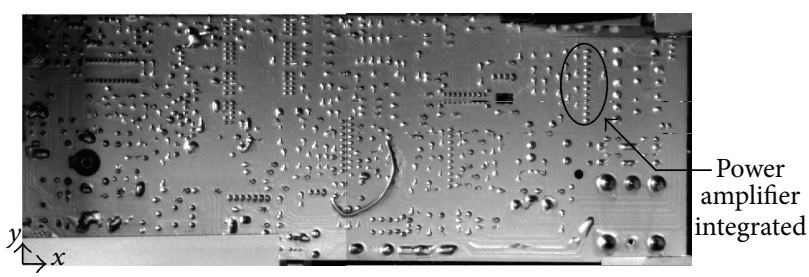

FIGURE 10: The coated backside PWB (the black ellipse indicates the power amplifier integrated).

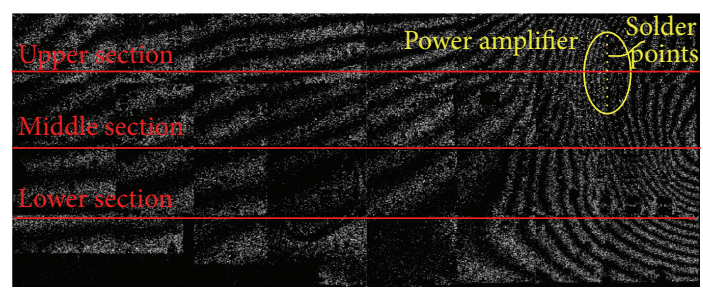

FIGURE 11: Whole $u$-displacement map of the PWB.

During the experiments, the electronic connections among different components remain unchanged, but the board has been taken out of the radio frame to expose its surface to the laser light, which has obviously underestimated the thermoelastic behavior in the real operation condition having less cooling transfer than that in the experiments. The board however has been mounted at the same constraint condition as in its operation.

A series of repeating experiments revealed the thermal steady state of the PWB reached in 15 minutes after operation started with about $90 \%$ of the maximum power available. Thus, in order to achieve a measurement of the whole field, the fringe patterns at 15 minutes after the starting point for each measuring zone in the PWB have been obtained as the steady state.

Figure 11 shows the speckle fringe pattern of the whole $u$ displacement field at the steady state from which the stress distribution is outlined. Images were processed with the state-of-the-art image processing software, Holo Moiré Strain Analyzer (HMSA), developed by C. A. Sciammarella and his collaborators [15]. It is clear to see that the stress distribution is not very uniform over the board. The stress concentration on the upper right hand side is due to the settlement of the power amplifier integrated at that zone as shown in Figure 10 


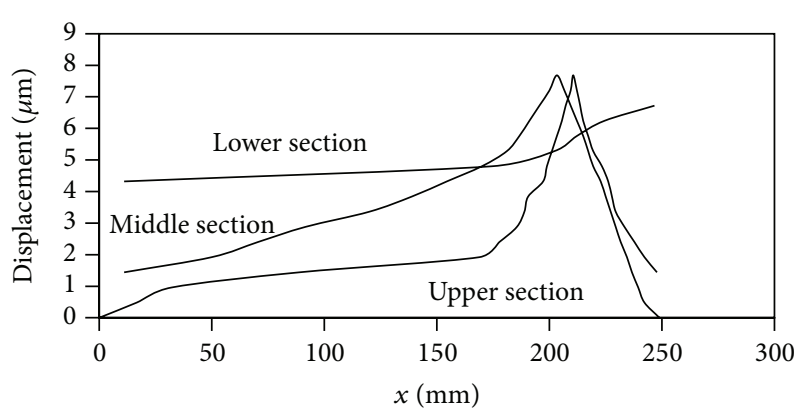

Figure 12: Displacement distribution in $x$ direction measured for the PWB.

and due to the fact that the moments and forces anchoring these high thermally stressed solder joints act on the board.

This stress concentration has a clear tension/compression characteristic since the fringes are nearly vertical while in other locations the fringes are almost horizontal which indicates the shear stress predomination. Such nonuniform stress distribution gives a very inconvenient failure time due to the breaking of local printed wires and solder joints.

The elastic modulus of common polyimide boards is about $2.7 \mathrm{GPa}$ and the Poisson ration is between 0.23 and 0.35 , while the elastic modulus of copper is about $124 \mathrm{GPa}$ [1]. The sensitivity of speckle interferometer was about $0.48 \mu \mathrm{m}$. Distributions of $u$-displacements along three control sections (namely, "upper," "middle," and "lower") are reported in Figure 12. It can be seen that the displacement gradient increases sharply near the location of the power amplifier.

Based on the above measurement, a simplified $x$ direction stress calculation is given here for the stress concentration zone. As correlation fringes are mainly oriented in the vertical direction, it can be supposed that strains in the $x$ direction predominate. Therefore, (4) can be simplified as follows:

$$
\sigma_{x x}=\frac{E(1-v) \varepsilon_{x x}}{(1+v)(1-2 v)} .
$$

For the board material, the strain $\varepsilon_{x x}$ is calculated by (7) as $0.45 \times 10^{-3}$. This value represents the frequency of the correlation fringes in correspondence with the solder points of the power amplifier (see dotted/etched lines in Figures 11 and 12). Taking $E=2.7 \mathrm{GPa}$ and $v=0.3$ for the board and $E=124 \mathrm{GPa}$ and $v=0.3$ for the printed copper wires in the region of interest (i.e., where the power amplifier is soldered onto the board), the $x$ direction stresses are 3.4 MPa for the board and $75 \mathrm{MPa}$ for the wires. These stresses are lower than those measured in the $y$ direction as the soldering develops right in the vertical direction, thus limiting the deformation and increasing the reaction forces.

\section{Conclusions}

From the experimental results, the very good applicability of the speckle technique for the problems studied has been demonstrated. The displacement map of the whole field has been obtained and can be taken as a verification during the electronic packaging design stages. High stress values are evident in the constraint points of the single components and in the location of the power component on the PWB. The stress state is strongly connected to the constraint reactions and also to the thermal disposal. For these reasons, in order to have a good design, it is very important to consider these two above-mentioned factors, so as to avoid probable and dangerous cracks in the structures. Considering that the wires are subjected to cyclic thermal loading, the design of the board should be also done very carefully to take into account possible fatigue failure.

Besides classical information on displacements and strains available from speckle measurements, we also determined stress concentrations at critical locations. This is an interesting improvement with respect to other studies on thermomechanical behavior of electronic packaging [16-19].

\section{Conflict of Interests}

The authors declare that there is no conflict of interests regarding the publication of this paper.

\section{References}

[1] P. A. Engel, Structural Analysis of Printed Circuit Board Systems, Springer, Berlin, Germany, 1993.

[2] T. P. Ma, Stresses in $\mathrm{SiO}_{2}$-on-Si Structures, vol. 4 of EMIS Datareviews, 1987.

[3] B. Han, D. Barker, and R. Mahaian, "Experimental/numerical mechanics in electronic packaging," in Proceedings of the 8th International Congress on Experimental Mechanics, vol. 1, Nashville,Tenn, USA, June 1996.

[4] C. Pappalettere, G. Rizzo, W. M. Sun, and B. Trentadue, "Thermal stress determination in a transistor by speckle interferometry," in Proceedings of the 8th International Congress on Experimental Mechanics, vol. 1, Nashville, Tenn, USA, June 1996.

[5] J. C. Dainty and J. W. Goodman, Laser Speckle, Springer, New York, NY, USA, 1984.

[6] R. Jones and C. Wykes, Holographic and Speckle Interferometry, Cambridge University Press, Cambridge, UK, 1989.

[7] M. Francon, Laser Speckle and Application in Optics, Academic Press, New York, NY, USA, 1979.

[8] L. Pagnotta and A. Poggialini, "Speckle pattern correlation methods: performance analysis by numerical simulation," in Proceedings of the International Conference on Materials Engineering, Gallipoli, Italy, 1996.

[9] P. R. Slangen, L. Berwart, C. de Veuster, J.-C. Golinval, and Y. F. Lion, "Digital speckle pattern interferometry (DSPI): a fast procedure to detect and measure vibration mode shapes," in First International Conference on Vibration Measurements by Laser Techniques: Advances and Applications, vol. 2358 of Proceedings of SPIE, Ancona, Italy, October 1994.

[10] C. A. Sciammarella and F. M. Sciammarella, Experimental Mechanics of Solids, John Wiley \& Sons, 2012.

[11] D. W. Robinson and D. C. Williams, "Digital phase stepping speckle interferometry," Optics Communications, vol. 57, no. 1, pp. 26-30, 1986.

[12] K. Creath, "Phase-shifting speckle interferometry," Applied Optics, vol. 24, no. 18, pp. 3053-3058, 1985. 
[13] M. Kujawinska, Interferogram Analysis, Institute of Physics Publishing, Philadelphia, Pa, USA, 1993.

[14] P. Jacquot, "Speckle interferometry: a review of the principal methods in use for experimental mechanics applications," Strain, vol. 44, no. 1, pp. 57-69, 2008.

[15] General Stress Optics, Holo-Moiré Strain Analyzer (HoloStrain) Version 2.0, General Stress Optics, Chicago, Ill, USA, 2008.

[16] K. Genovese, L. Lamberti, and C. Pappalettere, "A comprehensive ESPI based system for combined measurement of shape and deformation of electronic components," Optics and Lasers in Engineering, vol. 42, no. 5, pp. 543-562, 2004.

[17] F. Su, L. Liu, and T. Wang, "Evaluation of residual stress in flip chip using 3-D optical interferometry/FEM hybrid technique," Strain, vol. 43, no. 4, pp. 289-298, 2007.

[18] H. R. Schubach, "Investigation of the thermal deformation of electronic packages," in Experimental Analysis of Nano and Engineering Materials and Structures, E. E. Gdoutos, Ed., Springer, Dordrecht, The Netherlands, 2007.

[19] C. Casavola, L. Lamberti, V. Moramarco, G. Pappalettera, and C. Pappalettere, "Experimental analysis of thermo-mechanical behaviour of electronic components with speckle interferometry," Strain, vol. 49, no. 6, pp. 497-506, 2013. 

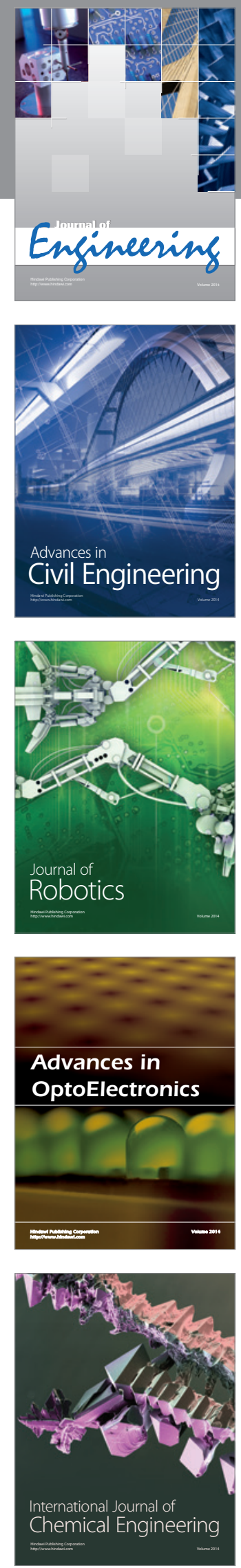

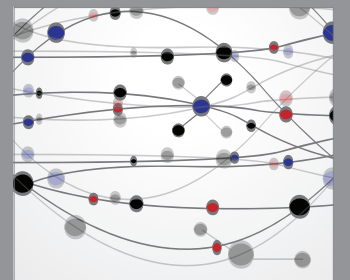

The Scientific World Journal
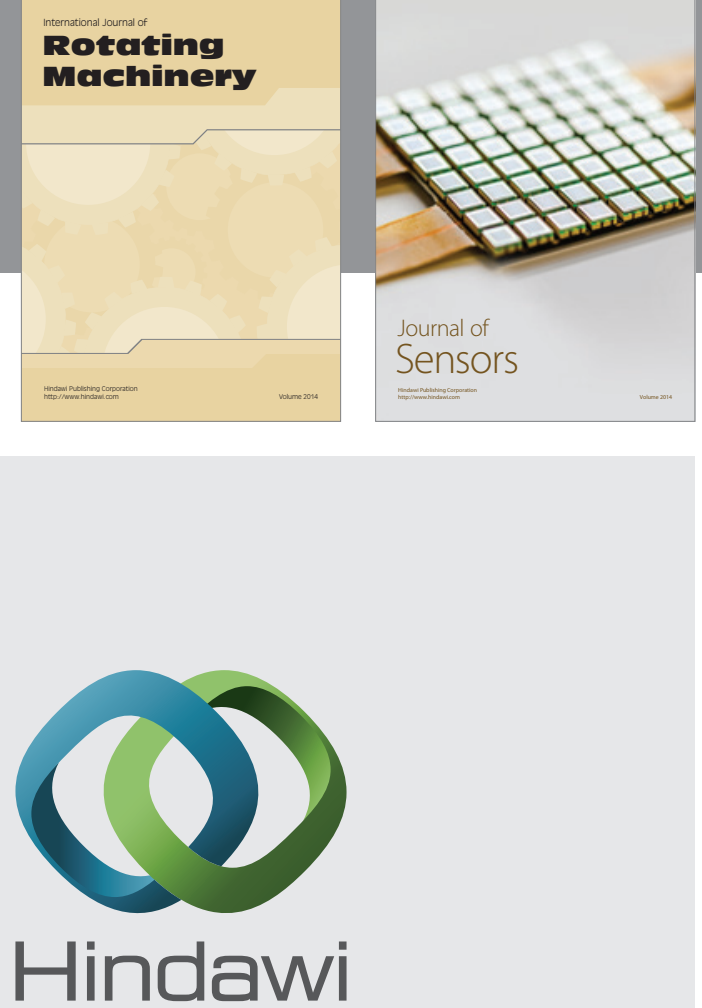

Submit your manuscripts at http://www.hindawi.com
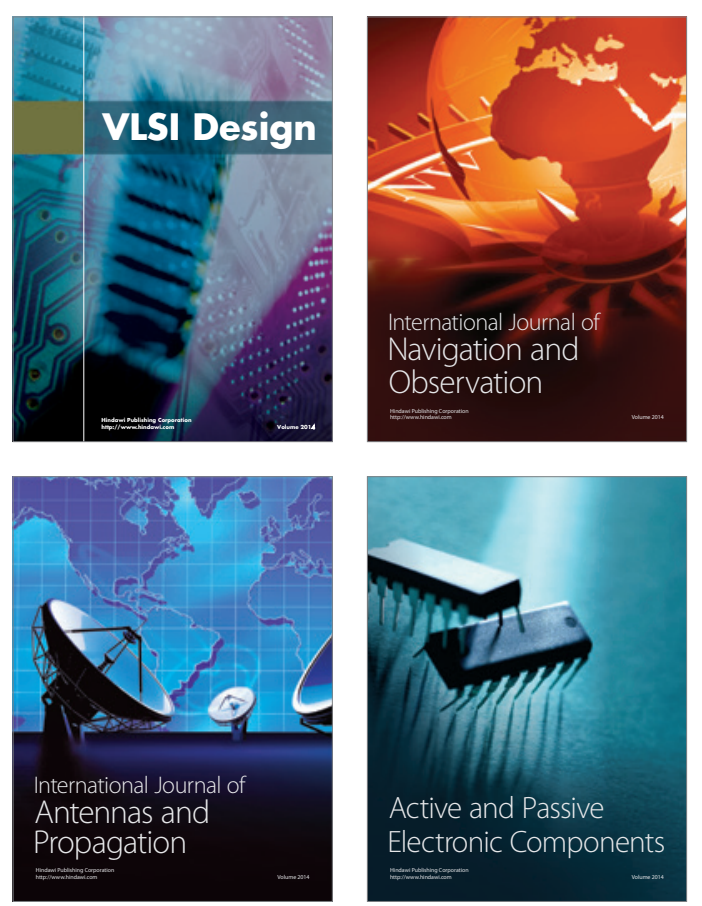
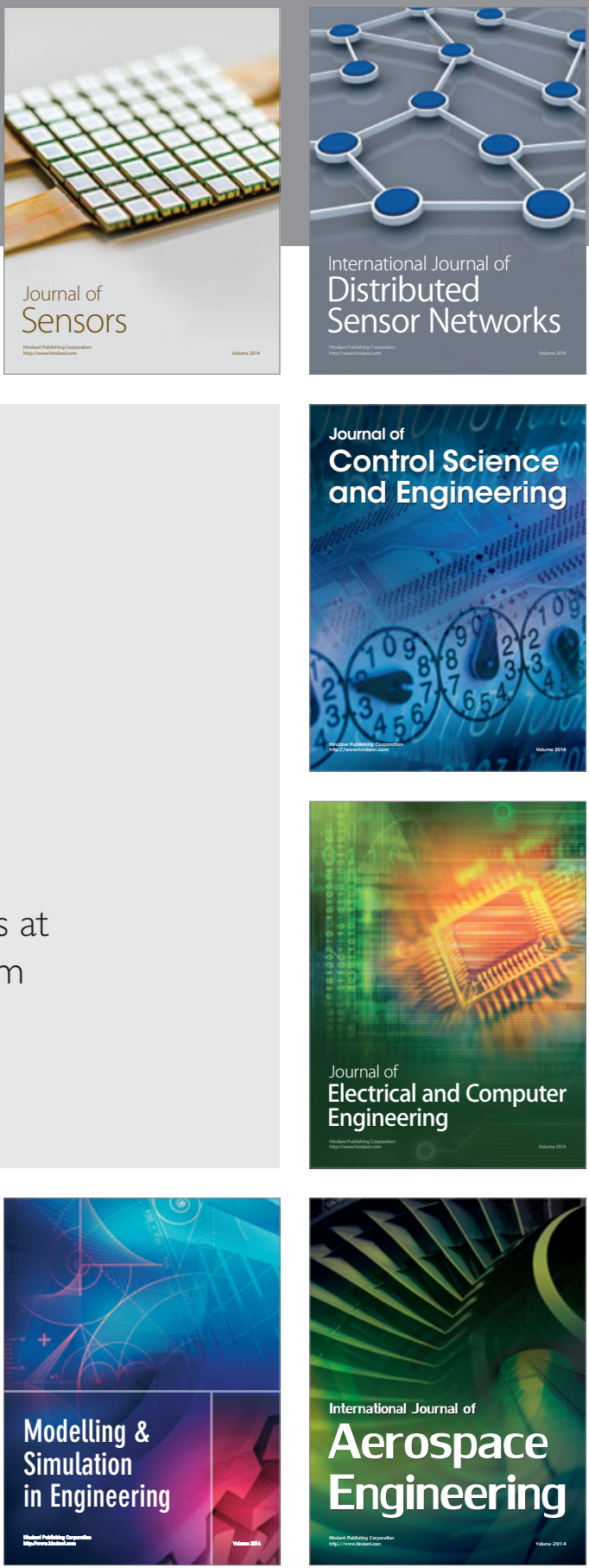

Journal of

Control Science

and Engineering
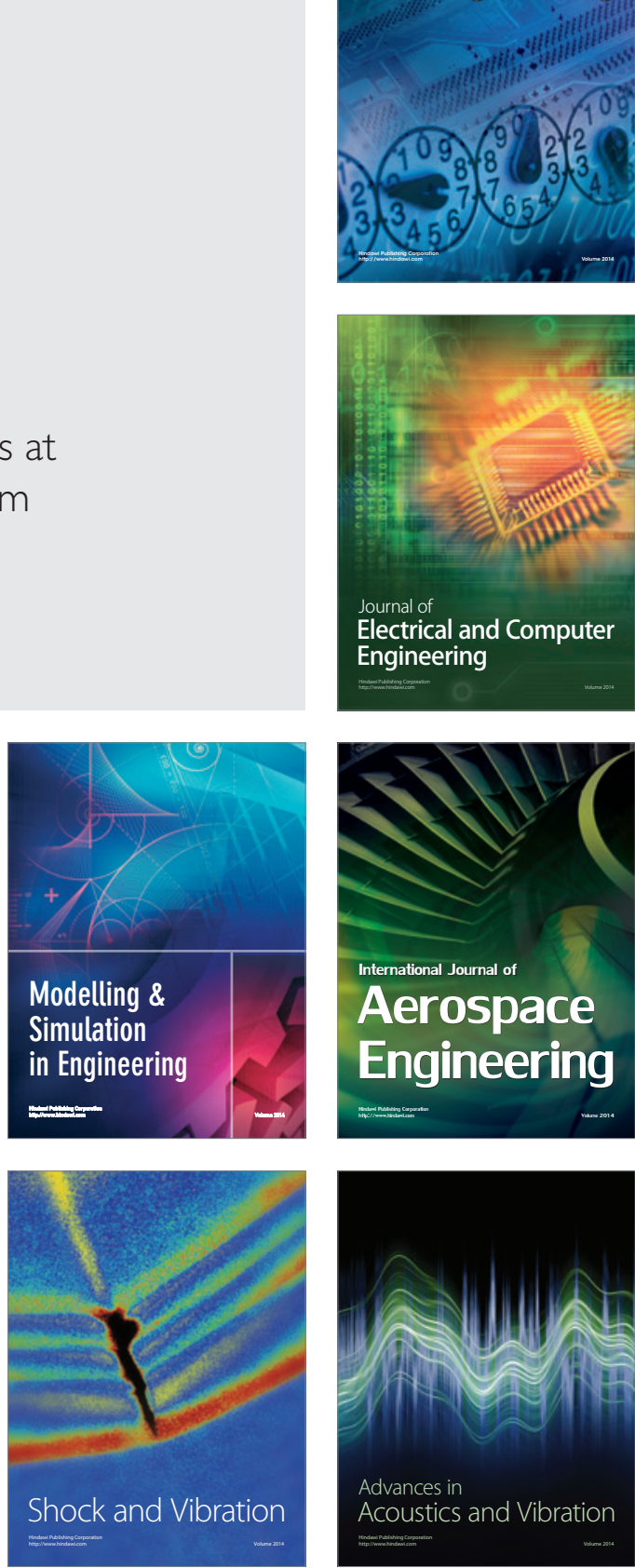